\title{
NONCLASSICAL AG PRESENTATION
}

Wednesday, July 8

W.18. Mucosal and Systemic Immune Response of Orally Delivered PsaA Subunit Antigen Microspheres in Mice

Simon Paulos ${ }^{1}$, Dilip Devineni ${ }^{1}$, Daniel Ezekwudo ${ }^{1}$, Susan Hollingshead ${ }^{3}$, Jacquelyn Sampson ${ }^{2}$, David Briles ${ }^{3}$, Edwin Ades $^{2}$, Ravi Palaniappan ${ }^{1}$

${ }^{1}$ Mercer University, Atlanta, GA; ${ }^{2}$ Center for Disease Control and Prevention, Atlanta, GA; ${ }^{3}$ University of Alabama at Birmingham, Atlanta, GA

Stimulating the mucosal immunity is of great interest in preventing pneumonia, which mainly invades the body via the upper respiratory tract. In this work, we have shown that a Streptococcus pneumoniae surface antigen PsaA has the potential to develop immunity in mice models after oral delivery when given in a microsphere formulation targeted to Peyer's patches. The microsphere formulation is designed to help overcome the harsh stomach environment and was optimized for size and charge and administered by oral route by gavage to $\mathrm{BALB} / \mathrm{c}$ mice following which systemic and mucosal immune responses were evaluated. Elevated serum antibody levels of IgG2a and IgA were detected in the group which received oral antigen entrapped polycaprolactone-maltodextrin microspheres versus naked protein antigens upon challenge with a pneumococcal bacterial strain (carriage moel EF3030). Additionally, cytokine responses upon ex-vivo re-stimulation of cells from lymphoid organs (Nasal Tract, Cervical Lymph Nodes, Lung and Spleen) with PsaA antigen were evaluated for IL-2, IL-4, IL-6, IFN- $\gamma$ and TNF- $\alpha$. High levels of IL-2, TNF- $\alpha$ and IFN- $\gamma$ were detected in the antigen re-stimulated lymphoid cells from orally administered antigen formulation, which correlated with the PsaA signature serum antibody response of IgG2a. 